\title{
Further Validation and Development of the Movement Imagery Questionnaire
}

\section{Sarah E. Williams, ${ }^{1}$ Jennifer Cumming, ${ }^{1}$ Nikos Ntoumanis, ${ }^{1}$ Sanna M. Nordin-Bates, ${ }^{2}$ Richard Ramsey, ${ }^{3}$ and Craig Hall ${ }^{4}$}

${ }^{1}$ University of Birmingham; ${ }^{2}$ Trinity Laban Conservatoire of Music and Dance; ${ }^{3}$ Université Catholique de Louvain; ${ }^{4}$ University of Western Ontario

\begin{abstract}
This research validated and extended the Movement Imagery QuestionnaireRevised (MIQ-R; Hall \& Martin, 1997). Study 1 ( $N=400)$ examined the MIQ-R's factor structure via multitrait-multimethod confirmatory factor analysis. The questionnaire was then modified in Study $2(N=370)$ to separately assess the ease of imaging external visual imagery and internal visual imagery, as well as kinesthetic imagery (termed the Movement Imagery Questionnaire-3; MIQ-3). Both Studies 1 and 2 found that a correlated-traits correlated-uniqueness model provided the best fit to the data, while displaying gender invariance and no significant differences in latent mean scores across gender. Study $3(N=97)$ demonstrated the MIQ-3's predictive validity revealing the relationships between imagery ability and observational learning use. Findings highlight the method effects that occur by assessing each type of imagery ability using the same four movements and demonstrate that better imagers report greater use of observational learning.
\end{abstract}

Keywords: ease of imaging, imagery ability, imagery perspective, observational learning, scale development

Imagery is a cognitive process that can play an important role in the planning and execution of movements or actions. It is frequently employed to aid motor skill learning, or relearning, as well as to improve motor performance in clinical, dance, and sport settings (for reviews, see Cumming \& Williams, 2012; Malouin, Richards, Jackson, \& Doyon, 2010; Page, 2010). Although imagery occurs in a number of sensory modalities (e.g., visual, auditory, olfactory), the focus of movement imagery is usually on visual and kinesthetic imagery, which are often experienced simultaneously (e.g., Cumming \& Ste-Marie, 2001). The effectiveness of imagery as a performance-enhancing strategy is dependent on the individual's ability to

Sarah E. Williams, Jennifer Cumming, and Nikos Ntoumanis are with the School of Sport and Exercise Sciences, University of Birmingham, Edgbaston, Birmingham, U.K. Sanna M. Nordin-Bates is now with the Swedish School of Sport and Health Sciences, Stockholm, Sweden. Richard Ramsey is now with the Wales Institute of Cognitive Neuroscience, School of Psychology, Bangor University, Bangor, Gwynedd, U.K. Craig Hall is with the School of Kinesiology, University of Western Ontario, London, Ontario, Canada. 
generate and control vivid images (Martin, Moritz, \& Hall, 1999). Individuals with greater imagery ability have been shown to outperform their lesser counterparts (e.g., Goss, Hall, Buckolz, \& Fishburne, 1986). Consequently, researchers often screen potential participants for their imagery ability before experiments and interventions (Cumming \& Ramsey, 2009).

A comprehensive yet inexpensive method of screening participants' visual and kinesthetic imagery abilities is the use of self-report questionnaires. One of the most popular and commonly used questionnaires is the revised version of the Movement Imagery Questionnaire (MIQ-R; Hall \& Martin, 1997). A briefer version of its predecessor, the Movement Imagery Questionnaire (MIQ; Hall \& Pongrac, 1983), the MIQ-R assesses the ability to mentally see and feel four simple movements: a knee lift, jump, arm movement, and waist bend. Participants image each movement twice: once before rating how easy it is to see the movement just imaged, and once before rating how easy it is to feel the movement just imaged. Ratings range from "very hard to see/feel" to "very easy to see/feel." The popularity of the MIQ-R led to a more recent version of the questionnaire (the MIQ-RS; Gregg, Hall, \& Butler, 2010) being developed specifically for use in the rehabilitation setting.

The different versions of the MIQ take the unique approach of instructing individuals to physically perform each movement before generating an image of that same movement. This is in contrast to other imagery ability questionnaires, such as the Vividness of Movement Imagery Questionaire-2 (VMIQ-2; Roberts, Callow, Hardy, Markland \& Bringer, 2008). Specific instructions are also provided for how each movement should be performed and imaged. Without such clear instructions, Caliari (2008) warns of considerable variability between individuals in what mental representation is produced. If a group of individuals were told to simply image kicking a ball (an example item from the VMIQ-2), different interpretations of these instructions might occur depending on personal experience. For example, one person might image kicking a rugby ball in the air, whereas another might image a soccer ball kicked along the ground. Further, the actual kicking action might vary from person to person, with some electing to image kicking the ball out of their hands, and others might image the initiation of the kick when the ball is positioned on the floor. Instructing participants to first perform the movement is likely to help reduce such discrepancies in imagery content-a factor known to influence reported imagery ability (Williams \& Cumming, 2011). This procedure also provides the questionnaire administrator with an opportunity to visually confirm whether participants correctly understand the desired movement before it is imaged. Of course, owing to its internal nature, only the imager is able to experience and evaluate the actual image.

Another reason to ask participants to physically perform the movement before imaging is to account for recency effects that might influence their imagery ability. How easily a movement is imaged might be affected by whether this movement was performed recently or frequently by the participant (Lequerica, Rapport, Axelrod, Telmet, \& Whitman, 2002). Returning to the ball kicking example, someone who regularly plays soccer will likely recall this experience more readily from memory than an individual who has not performed this action for themselves in a long time, or ever. Differences in how easily these two participants are able to image ball kicking might therefore be influenced by their experience with the task. Physical performance before imaging reduces this problem by ensuring each participant is 
able to readily recall the movements. Consequently, the resulting scores are more likely to be an accurate reflection of the participants' actual ability to image rather than how recently the movement was physically performed.

Another MIQ-R strength is that participants perform all images in the starting positions adopted when physically performing the movements. This increases the functional equivalence at the neural level between the image and execution of the movement and incorporates the physical element of the PETTLEP model-something that many imagery interventions do (e.g., Robin et al., 2007; Smith et al., 2007). Therefore, the MIQ-R is likely to provide a more accurate assessment of participants' imagery ability compared with questionnaires completed while seated, as the images are likely to be performed more similarly to how most intervention imagery is performed.

Further to these strengths of the questionnaire, good internal reliability has consistently been reported for the MIQ-R subscales (e.g., Abma, Fry, Li, \& Relyea, 2002; Vadocz, Hall, \& Moritz, 1997), and a handful of studies have demonstrated its predictive validity (e.g., Vadocz et al., 1997). However, evidence of the MIQ-R's factorial validity is currently lacking, with the majority of existing studies having been conducted on the original MIQ (e.g., Atienza, Balaguer, \& Garcia-Merita, 1994). Compared with the MIQ, the MIQ-R has fewer items and the rating scale is reversed. Therefore, it cannot be assumed that they demonstrate a similar factor structure and model fit.

Until very recently, research had not investigated the psychometric properties of the commonly used English version of the MIQ-R. To address this gap, Monsma, Short, Hall, Gregg, and Sullivan (2009) confirmed its factor structure with structural equation modeling, tested for gender invariance (i.e., tested whether the model fit varied between males and females), and examined internal and temporal reliability. As for the MIQ, the MIQ-R was found to have good internal and temporal reliability, with Cronbach alpha coefficients of .84 and .88 for the visual and kinesthetic subscales, respectively, and test-retest reliability coefficients of .80 for visual imagery and .81 for kinesthetic imagery. The confirmatory factor analysis (CFA) showed a poor fit to the data for the hypothesized factor structure of the MIQ-R (comparative fit index $=.90$, Bentler-Bonett non-normed fit index $=.91$, standardized root mean square residual $=.28$, and root mean square error of approximation $=.15$ ). However, once a path was inserted in the model suggesting a relationship between the visual and kinesthetic subscales, the model fit significantly improved (comparative fit index $=.99$, Bentler-Bonett non-normed fit index $=.98$, standardized root mean square residual $=.03$, and root mean square error of approximation $=.06$ ). This finding was contrary to the earlier validation of the French version of the MIQ-R (Lorant \& Nicholas, 2004), in which visual and kinesthetic modalities were found to be separate, unrelated constructs, but is keeping with the more typical relationship found. Because studies frequently employ versions of the MIQ-R to assess the visual and kinesthetic imagery ability of both males and females (e.g., Guillot et al., 2009; Williams, Cumming, \& Edwards, 2011), it is perhaps surprising that the MIQ-R factor structure was not invariant across gender. That is, the model displayed a good fit to the female sample, but data on the male sample failed to converge to the model (Monsma et al., 2009). Although two independent $t$ tests revealed that males and females did not significantly differ from one another in their visual and kinesthetic imagery ability scores, due to the 
gender variance in model fit, this finding may be influenced by the questionnaire's varying factor structure.

Although a promising step in providing evidence of the MIQ-R's psychometric properties, Monsma et al. (2009) used a traditional CFA that did not allow them to consider the common variance that might exist by employing the same four movements to assess both visual and kinesthetic imagery (i.e., a method effect). For example, an individual's visual imagery ability of a waist bend is likely to be associated with his or her kinesthetic imagery ability of this movement. Multitraitmultimethod (MTMM) analyses offer a more appropriate statistical approach for establishing the relationship among the traits (i.e., visual and kinesthetic imagery ability) when the effects of method variance and random error are present (Schmitt \& Stults, 1986; Marsh \& Grayson, 1995). The absence of an MTMM approach in the previous CFAs of the MIQ-R might explain why inconsistent models have been produced (i.e., a two-factor correlated traits English version, and a two-factor uncorrelated traits French version). It might also be the reason why the previously established factor structure of the English version of the MIQ-R was not invariant between males and females as expected. Clarity regarding these issues is likely to be achieved through a more extensive CFA investigation using an MTMM approach. Testing and comparing a number of models will identify the most appropriate model fit for MIQ-R data (Marsh, 1989), and provide further support for its use as a measure of movement imagery ability. From a conceptual point of view, comparing these models will provide further insight into imagery ability as a reflection of the imagery process. Specifically, it will investigate whether individuals' ease of imaging specific content from one modality is related to their capacity to image the same content from another modality. As part of the ongoing validation of the questionnaire, it is also necessary to examine other forms of validity, including predictive validity, and more contemporary methods of establishing internal reliability. We address these issues in three separate studies.

\section{Study 1}

The aim of the first study was threefold. First to investigate whether a model using an MTMM approach to CFA provided a better fit to the data than a first-order CFA, which does not account for potential method effects caused by assessing visual and kinesthetic movement imagery ability using four movements. The second aim was to determine whether a correlated or uncorrelated traits model provided the best fit to the data, attempting to resolve the ambiguity of previous MIQ-R validation (Lorant \& Nicholas, 2004; Monsma et al., 2009). Once the best fitting model was established, the third aim was to reexamine the MIQ-R's suitability of assessing male and female movement imagery ability by using two separate approaches. The first was to test for gender invariance, and the second was to investigate whether significant differences existed in the latent mean structures between males and females. The latter is an analysis that has yet to be done in the process of validating the MIQ-R or any other measure of imagery ability.

It was hypothesized that, owing to the same movements being used to assess both traits (i.e., visual imagery and kinesthetic imagery), an MTMM CFA model would display a better fit to the data than a CFA, which does not account for method effects. Based on the validation of the VMIQ-2 (Roberts et al., 2008), which also 
assesses multiple dimensions of imagery ability using the same items, and previous research that demonstrates significant correlations between visual and kinesthetic imagery (e.g., Abma et al., 2002; Goss et al., 1986; Vadocz et al., 1997), we hypothesized that a correlated trait-correlated uniqueness model would provide the best fit to the data. In addition, it was hypothesized that our final model would display gender invariance, and based on studies demonstrating no gender differences in imagery ability (e.g., Lorant \& Nicholas, 2004; Monsma et al., 2009; Williams et al., 2011), that there would be no significant gender differences in latent means.

\section{Method}

\section{Participants}

Four hundred males $(n=181)$ and females $(n=219)$ with a mean age of 20.83 years $(S D=2.14)$ participated in the study. Participants were a combination of student athletes and dancers who were all physically capable of performing the four MIQ-R movements and had no previous imagery training.

\section{Measures}

Demographic Information. Participants provided information regarding their age and gender.

Movement Imagery Questionnaire-Revised (MIQ-R). The MIQ-R (Hall \& Martin, 1997) is an eight-item questionnaire assessing movement imagery ability of four basic movements: a knee lift, jump, arm movement, and waist bend. Ease of imaging is measured in both visual and kinesthetic modalities. For each item, participants read a description of the movement. They then physically perform the movement before assuming the same starting position to either visually or kinesthetically image the movement. Following this step, participants rate their ease of imaging on a 7-point Likert-type scale ranging from 1 (very hard to see/feel) to 7 (very easy to see/feel). After the items for each subscale are averaged, a higher score represents a greater ease of imaging. Internal reliability was assessed using composite reliability (CR) and average variance extracted (AVE) due to the limitations associated with Cronbach's alpha. The criterion level was set at the values of .70 and .50 respectively (Hair, Anderson, Tatham, \& Black, 1998). Both subscales demonstrated adequate CR: visual imagery $=.88$, and kinesthetic imagery $=.82$, and AVE: visual imagery $=.65$, and kinesthetic imagery $=.53$.

\section{Procedures}

Following ethical approval, participants were recruited from the university where the lead author is based, with some participants receiving partial fulfillment of course credits. Those interested in taking part were provided with an information sheet and the nature of the study was explained in more detail by an investigator. Those willing to participate signed a consent form and completed the MIQ-R either in small groups or individually. Once completed, all forms were returned to the investigator and participants were thanked for their participation, which took no more than 10 min. 


\section{Data Analysis}

All data were screened for univariate outliers through the examination of item skewness and kurtosis values. Multivariate normality was examined using Mardia's coefficient (Mardia, 1970). When data were identified as non-normal, the bootstrapping technique was employed in all further analyses. Bootstrapping enables the creation of multiple subsamples from the original data with parameter distributions being subsequently examined in each of these samples (Byrne, 2009).

An MTMM approach to CFA was used to establish the relationship among the traits (i.e., visual and kinesthetic imagery) when method variance effects and random error are present (Schmitt \& Stults, 1986). Convergent and discriminant validity were also assessed, with large factor loadings on trait factors supporting convergent validity, and large correlations between trait factors suggesting lack of discriminant validity among traits (Byrne, 2009). Selection of the most appropriate model depended on which displayed the best fit indices and whether the model converged to a proper solution (Marsh \& Grayson, 1995). Failure to converge or convergence to an improper solution was not considered creditable. Once the model with the best fit was selected, multisample analysis was conducted to examine whether the factor structure was sustained for both males and females. Finally, we investigated whether there were gender differences in the latent means of the factors (i.e., visual imagery and kinesthetic imagery).

Multitrait-multimethod analysis, gender invariance, and latent mean structure testing were conducted via structural equation modeling with maximum likelihood estimations using AMOS 16.0 (Arbuckle, 2007). Each model's overall goodness of fit to the data were examined and determined using the chi-squared likelihood ratio statistic ratio $\left(\chi^{2}\right)$. Although a nonsignificant $\chi^{2}$ value represents a good model fit, this is not often observed in practice (MacCallum, 2003). Although this value should not be disregarded, the Tucker Lewis index (TLI), comparative fit index (CFI), standardized root mean square residual (SRMR), and root mean square error of approximation (RMSEA) were also used to provide additional information regarding the adequacy of the model fit. Hu and Bentler (1999) suggest that values of close to .95 or above indicate a relatively good fit for the TLI and CFI, and values close to .08 or lower and .06 or lower indicate relatively good fit for the SRMR and RMSEA respectively. Although there is some debate in the literature regarding how appropriate the values indicative of adequate model fit are (see Markland, 2007), these criteria are still the most commonly reported as indications of an adequate model fit when validating questionnaires. Finally, standardized factor loadings, standardized residuals, and modification indices were examined to investigate any model misspecification.

For MTMM, Marsh (1989) suggests that four models should be tested and compared with determine the best model fit. These are the correlated trait (CT) model, the correlated trait-correlated method model, the correlated trait-uncorrelated method model, and the correlated trait-correlated uniqueness (CTCU) model. As can be seen in Figure 1, the correlated trait-correlated method model involves correlating both traits, as well as the four methods (represented by the dashed line components of the model). The correlated trait-uncorrelated method model allows both traits to be correlated but the four methods are not (consequently, the dashed line correlations between each method would be removed). The CT model has no method effects, meaning that all the dashed line components of Figure 1 would not be present. This CT model is equivalent to that tested by Monsma et al. (2009) during their MIQ-R validation. 


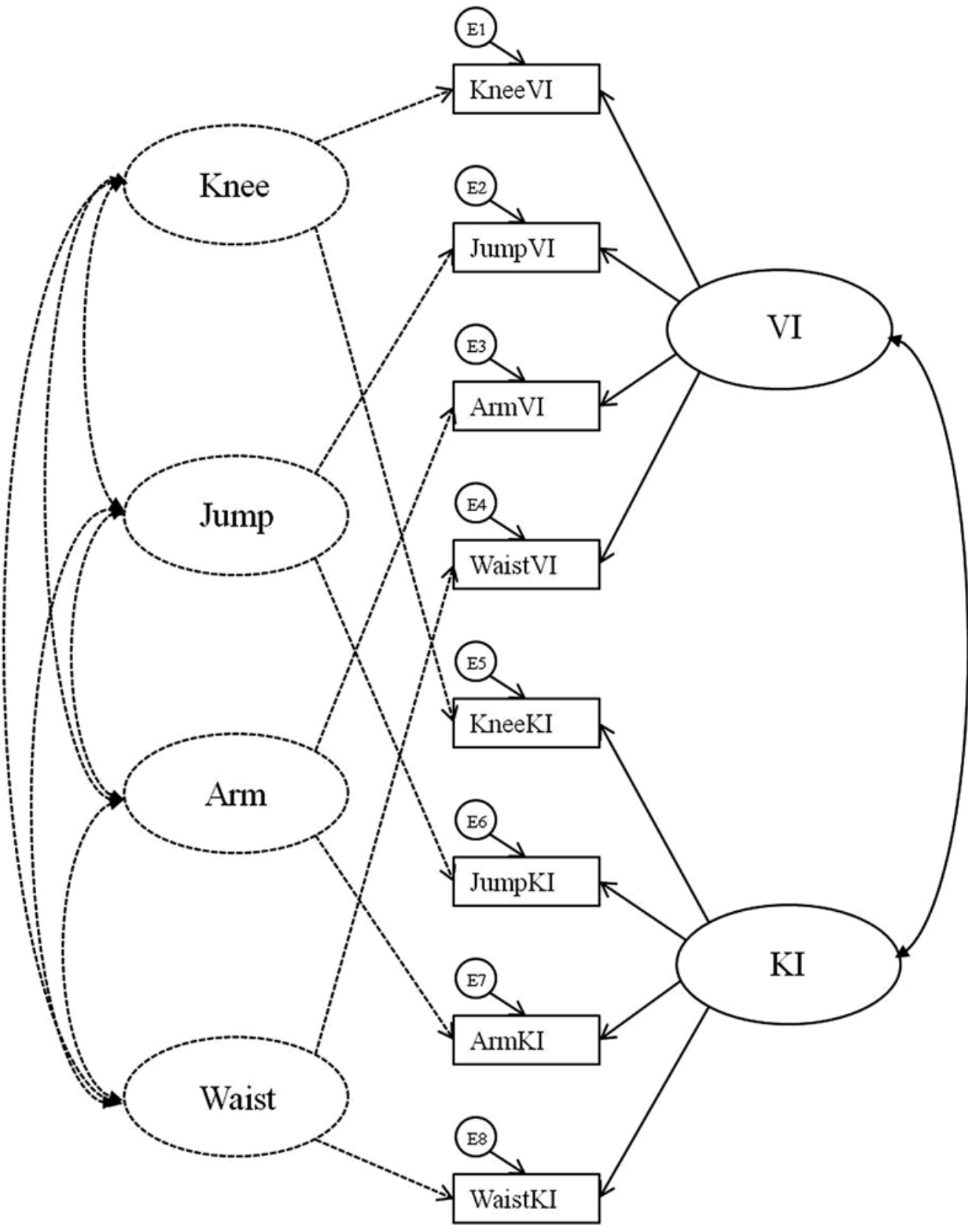

Figure 1 - Hypothesized multitrait-multimethod correlated trait-correlated method model. $\mathrm{VI}=$ visual imagery. $\mathrm{KI}=$ kinesthetic imagery . 
The CTCU model postulates that both imagery types are correlated and the method effects are obtained from correlated uniqueness (i.e., correlated error terms) among the responses that share the same method. Therefore, the dashed line components of the model would be removed and lines between each error term of the same movement would be inserted (e.g., between E1 and E5). Marsh (1989) explains that the size of correlations between the uniqueness terms and the fit of this model compared with that of the CT model determine the extent of method effects. Further, comparing the CTCU model with the correlated trait-correlated method and correlated trait-uncorrelated method models tests whether any method effects are multidimensional or unidimensional. While the correlated trait-correlated method and correlated trait-uncorrelated method models both assume that method effects are unidimensional (i.e., they are explained by one latent method factor), the CTCU model does not have this assumption, instead assuming they are multidimensional. For a more comprehensive description of MTMM analysis, please see the following sources: Kenny and Kashy (1992) and Marsh (1989).

\section{Results}

\section{Data Screening and Normality}

All item skewness and kurtosis values were distributed within the tolerance levels of normality assumptions. Mardia's coefficient (Mardia, 1970) revealed that data did not display multivariate normality (normalized estimate $=6.98$ ); therefore, bootstrapping was employed in all further analysis.

\section{MTMM CFA}

The correlated trait-correlated method and correlated trait-uncorrelated method models yielded improper solutions. Both displayed negative variances and were therefore disregarded. The other two models (CT and CTCU) resulted in proper solutions and consequently their fit indices were examined (see Table 1). Standardized

\section{Table 1 MIQ-R and MIQ-3 Multitrait-Multimethod Confirmatory Factor Analysis Goodness-of-Fit Indices for the Models With a Proper Solution}

\begin{tabular}{lcccccccc}
\hline Model & $\chi^{2}$ & $\boldsymbol{d} \boldsymbol{f}$ & TLI & CFI & SRMR & $\begin{array}{c}\text { RMSEA } \\
(\mathbf{9 0} \% \mathbf{C l})\end{array}$ & $\Delta \chi^{2}$ & $\Delta \boldsymbol{d f}$ \\
\hline Study 1 & & & & & & & & \\
$\quad$ (1) CT & $37.35 * *$ & 19 & .98 & .98 & .03 & $.05(.03-.07)$ & & \\
$\quad$ (4) CTCU & $25.99 *$ & 15 & .99 & .99 & .03 & $.04(.01-.07)$ & $11.36 *$ & 4 \\
Study 2 & & & & & & & & \\
$\quad$ (1) CT & $117.60 * *$ & 51 & .95 & .96 & .04 & $.06(.05-.07)$ & & \\
(4) CTCU & $75.12^{* *}$ & 39 & .97 & .98 & .04 & $.05(.03-.07)$ & $42.48 * *$ & 12 \\
\hline
\end{tabular}

Note. The CT are correlated trait models from Studies 1 and 2, and the CTCU are correlated trait-correlated uniqueness models from Studies 1 and $2, \Delta \chi^{2}=$ chi-square difference from the CT model, $\Delta d f=$ difference in degrees of freedom from the CT model.

$* p<.05 . * * p<.01$. 
factor loadings ranged between 0.70 and 0.84 (CT model) and between 0.70 and 0.84 (CTCU model). The intertrait correlations were .25 for the CT model and .23 for the CTCU model. Finally, the uniqueness correlations for the CTCU model ranged between .03 and .11. Inspection of the modification indices and standardized residuals revealed all values were within acceptable limits and no offending estimates existed (Hair et al., 1998). Consequently, all items in both models meaningfully contributed to their intended subscales. Although the CT model also displayed a good fit to the data, inspection of the correlated error variances in the CTCU model revealed significant correlations with one another $(p<.05)$. The $\chi^{2}$ difference test was used to investigate the relative goodness of fit between the two models. Results revealed a significantly smaller $\chi^{2}$ value for the CTCU model, thus demonstrating it to provide the best fit to the data.

\section{Alternative Model}

Although results support the CTCU model, previous validation of the French version of the MIQ-R suggested that visual and kinesthetic imagery are separate constructs (Lorant \& Nicholas, 2004). Therefore, a model similar to the CTCU was tested, but the correlation between the traits (i.e., visual and kinesthetic imagery) was removed (uncorrelated trait-correlated uniqueness model). Results revealed a poorer model fit to the data, $\chi^{2}(16)=40.80, p=.001, \mathrm{CFI}=.97$, TLI $=.96$, SRMR $=.09$, RMSEA $=.06(90 \% \mathrm{CI}=.04-.09)$, which was confirmed to have a significantly larger $\chi^{2}$ value, $\Delta \chi^{2}=14.81, p<.001$.

\section{Gender Invariance}

Gender invariance of the CTCU model was conducted using a sequential testing approach via multisample CFA. A baseline model was established before four increasingly constrained models were tested. The first constrained the factor loadings to be equal across both gender groups, the second also constrained the factor variances, the third also constrained the factor covariances, and the fourth also constrained the error covariances (Byrne, 2009). The $\chi^{2}$ difference test was used to investigate the relative goodness of fit between increasingly constrained models. Based on the recommendations of Cheung and Rensvold (2002), we considered a change in CFI of $\leq .01$ to be reflective of model invariance. Goodness-of-fit results for the five models of the gender invariance analysis displayed good model fit and are reported in Table 2. In accordance with our hypothesis, the MIQ-R's factorial invariance across males and females was supported by the nonsignificant change in $\chi^{2}$, and the change in CFI being less than .01 between each increasingly constrained model.

\section{Latent Means}

Latent means analysis was also conducted on the CTCU model. Similar to the analysis of gender invariance, a baseline model was first established. Factor loadings and observed item means were then constrained equal across groups and error term means were constrained to 0. Finally, the factor means (unobserved means derived from the observed item means loading on the factor) of the female group were constrained to 0 to serve as the reference categories, whereas the male group 





factor means were freely estimated (Bentler, 1995). Thus, the results indicate whether the male latent mean scores significantly differ from female latent mean scores but do not report the actual latent male and female mean scores (Byrne, 2009). Inspection of the latent mean estimates for male participants revealed no significant differences in visual imagery $(-.033, p=.762)$ or kinesthetic imagery $(-.051, p=.618)$ compared with females. Goodness of fit results demonstrated that the model with constrained loadings and item intercepts displayed a good fit to the data, $\chi^{2}(42)=5.37, p=.086, \mathrm{CFI}=.99, \mathrm{TLI}=.99, \mathrm{SRMR}=.04, \mathrm{RMSEA}$ $=.03(90 \% \mathrm{CI}=0.01-0.05)$.

\section{Discussion}

Results of the MTMM CFA revealed that the CTCU model provided a significantly better fit to the data compared with the CT model. This was further supported by the significant correlated error variances between the same methods (e.g., between both knee lift items). This finding was in support of our hypothesis highlighting the influence that assessing both types of imagery ability using the same items can have on MIQ-R results-a consideration that has previously been overlooked. Validation of the VMIQ-2 also found a CTCU model to be a good fit to the data (Roberts et al., 2008). By comparing a correlated traits version of the model to an uncorrelated version, our results revealed that visual and kinesthetic imagery should be considered as separate but related constructs. This finding is in accordance with previous studies (Abma et al., 2002; Goss et al., 1986; Vadocz et al., 1997).

Unlike previous MIQ-R validation, our findings support the MIQ-R as a measure of movement imagery ability for both males and females, as the factor structure was invariant across gender. This contradicts previous findings in which the proposed model displayed a better fit for females compared with males (Monsma et al., 2009). This discrepancy may be because method effects were not considered in previous validations of the MIQ-R. There were also no significant differences in male and female latent mean scores. Although some studies have detected gender differences in imagery ability, this is usually regarding spatiovisual imagery ability (e.g., Campos, Pérez-Fabello, \& Gómez-Juncal, 2004). Our finding is in accordance with studies demonstrating no significant differences between males and females in ease of movement imagery (Lorant \& Nicholas, 2004; Monsma et al., 2009; Williams et al., 2011).

\section{Study 2}

Despite the MIQ-R being validated in Study 1, researchers have argued that this questionnaire is limited by its inability to distinguish between visual perspectives (e.g., Roberts et al., 2008), that is, whether the image is seen from an internal visual imagery perspective or an external visual perspective. An external visual imagery perspective, also known as third-person perspective, is when an individual views himself or herself from another person's perspective (Hall, 2001). By comparison, an internal visual imagery perspective, also known as first-person perspective, is when an individual views the image through their own eyes. It is what the individual would see if they were actually performing the movement 
(Hall, 2001). Research has demonstrated differences in neuron activation when imaging from a first-person perspective compared with a third-person perspective (Ruby \& Decety, 2001). In addition, differences in motor evoked potentials have been elicited through transcranial magnetic stimulation when performing motor imagery from an internal perspective compared with that from an external perspective (Fourkas, Avenanti, Urgesi, \& Aglioti, 2006). These differences in brain activation between an internal and external perspective suggest that both are distinct cognitive processes.

Both visual imagery perspectives are also proposed to serve unique benefits. An external visual imagery perspective is valuable when performing tasks such as the learning of movements, and when form or body coordination is important, as the imager is presented with a view of how the movement or action should be performed, such as limb positions (White \& Hardy, 1995). Internal visual imagery is thought to be beneficial for open skills when timing is important. From this internal position, the individual is able to rehearse spatial locations and at what time a movement should be initiated (White \& Hardy, 1995). Depending on the imagery content and the intended outcome, it has been suggested that a particular perspective may be most beneficial. Callow and Hardy (2004) have suggested that individuals may find it easier to see the form-based movements of the MIQ-R from an external visual imagery perspective. Further, some athletes prefer to image from one perspective more than another, whereas others prefer switching between the two (e.g., Cumming \& Ste-Marie, 2001) and altering their images to take advantage of different viewing angles (e.g., Callow \& Roberts, 2010).

Altogether, it appears logical and necessary that the MIQ-R be extended to more fully capture an individual's visual imagery ability. To this end, a second study was conducted to create and validate a modified version of the questionnaire, called the Movement Imagery Questionnaire-3 (MIQ-3), to separately assess external visual imagery, internal visual imagery, and kinesthetic imagery ability. The primary aim of Study 2 was to validate the MIQ-3 using the same MTMM CFA approach as in Study 1. A second aim was to compare the final three-factor model against alternate two-factor models to ensure that assessing external visual imagery, internal visual imagery, and kinesthetic imagery separately provided the best model fit. A third aim was to test the best fitting model for gender invariance and compare the latent mean structures of males and females to investigate any significant gender differences in external visual imagery, internal visual imagery, and kinesthetic imagery ability.

Finally, investigating the concurrent validity of the MIQ-3 was a fourth aim, by examining whether its subscales correlated with the subscales of the VMIQ-2. Although the VMIQ-2 assesses vividness and the MIQ-3 ease of imaging, it has been suggested that both dimensions reflect the processes of image formation, transformation, and maintenance (Roberts et al., 2008). As such, a relationship exists between how easily and vividly a movement is imaged; for example, a good imager will likely find it easy to vividly image performing a knee lift. However, these dimensions are not one and the same. Take, for example, the image of swinging on a rope (another item from the VMIQ-2): an individual who has little experience with this movement may find it more difficult to generate this image with the same level of vividness as another movement with which they are more experienced. Therefore, we argue for a conceptual distinction between ease and vividness. 
We hypothesized that, similar to Study 1, a CTCU model accounting for method effects would display the best fit to the data. Because external visual imagery, internal visual imagery, and kinesthetic imagery are considered separate but related constructs (e.g., Roberts et al., 2008), a second hypothesis was that a model comprising three imagery traits as separate factors would provide a better fit to the data than alternative models that force imagery perspectives onto the same factor. Again based on Study 1, we hypothesized that our final model would be gender invariant and there would be no significant differences in the latent mean structure scores between males and females. Finally, it was hypothesized that the MIQ-3 would separately assess the ability to image external visual imagery, internal visual imagery, and kinesthetic imagery and subsequently each subscale would significantly correlate with its respected subscale on the VMIQ-2. This hypothesis was based on significant correlations previously identified between the VMIQ-2 and the MIQ-R (Roberts et al., 2008).

\section{Method}

\section{Participants}

A new sample of 370 participants (male $=185$; female $=185)$ with a mean age of 20.29 years $(S D=2.25)$ took part in Study 2. Participants were all healthy student athletes capable of physically performing the four MIQ-R movements and had no previous imagery training.

\section{Measures}

Demographic Information. The measures were identical to Study 1.

Movement Imagery Questionnaire-3 (MIQ-3). The MIQ-3 is an adaption of the MIQ-R (Hall \& Martin, 1997) and is composed of three subscales assessing external visual imagery and internal visual imagery, as well as kinesthetic imagery. The same four movements are physically performed and imaged three times, creating a 12-item questionnaire. The rating scales from the MIQ-R were retained such that participants' responses varied from 1 (very hard to see/feel) to 7 (very easy to see/feel), with a higher average score for a subscale representing a greater ease of imaging. Participants were provided with definitions of external visual imagery, internal visual imagery, and kinesthetic imagery before they completed the questionnaire. These were based on definitions of external visual imagery, internal visual imagery, and kinesthetic imagery used within the imagery literature (e.g., Hall, 2001; Callow \& Waters, 2005). External visual imagery was defined as "when you watch yourself performing the movement from an outside point of view or third person perspective. It can be likened to watching yourself on television or from another person's perspective." Internal visual imagery was defined as "when you watch yourself performing the movement from an inside point of view or first person perspective. It is as if you were looking out through your own eyes whilst performing the movement and is therefore what you would see while actually doing the movement." Kinesthetic imagery was defined as "the feelings and sensations experienced if you were actually producing the movement. It includes things such as feeling your muscles contract or feeling an object your body makes contact 
with." The MIQ-3 demonstrated good internal reliability for each subscale, with CR values of .83 (external visual imagery), .79 (internal visual imagery), and .85 (kinesthetic imagery), and AVE values of .55 (external visual imagery), .52 (internal visual imagery), and .59 (kinesthetic imagery).

Vividness of Movement Imagery Questionnaire-2 (VMIQ-2). To complete the VMIQ-2, participants rate the vividness of 12 movements for each of the three subscales: external visual imagery, internal visual imagery, and kinesthetic imagery (36 items in total). Participants were instructed to first image all items using external visual imagery, followed by internal visual imagery, and kinesthetic imagery. Movements include specific actions such as "throwing a stone into water" and whole-body movements such as "running up stairs." Each image was rated on a 5-point Likert-type scale, ranging from 1 (Perfectly clear and as vivid as normal vision or feel of movement) to 5 (No image at all, you only "know" that you are thinking of the skill). For easier comparison with the MIQ-3, the ratings were reverse scored such that a higher score represented a more vivid image. The VMIQ-2 has been shown to be a valid and reliable questionnaire (Roberts et al., 2008). In the current study the VMIQ-2 demonstrated good internal reliability for each subscale with CR values of .94 (external visual imagery), .93 (internal visual imagery), and .93 (kinesthetic imagery), and AVE values of .56 (external visual imagery), .52 (internal visual imagery), and .53 (kinesthetic imagery).

\section{Procedures}

The procedures were identical to Study 1 with the exception that a subsample of participants $(n=168)$ also completed the VMIQ-2. Questionnaires were completed in a counterbalanced order to eliminate order effects.

\section{Data Analysis}

Data were screened for outliers using the same procedures as in Study 1. Once the best three-factor model was selected using MTMM CFA, this model was compared with two alternative two-factor models to determine whether a three-factor model provided the best fit to the data. Gender invariance and latent means structures analyses were then conducted as in Study 1. The same computer package, bootstrapping technique, multivariate normality test, and goodness-of-fit criteria used in Study 1 were employed. Concurrent validity was established by examining the covariance between the MIQ-3 and VMIQ-2's external visual imagery, internal visual imagery, and kinesthetic imagery subscales in a measurement model. The factor structure of the each questionnaire was first examined (Kline, 2005) before the model as a whole was tested and covariance between each subscale investigated.

\section{Results}

\section{Data Screening and Normality}

Mardia's coefficient (Mardia, 1970) revealed that the data did not display multivariate normality (normalized estimate $=11.64$ ), and bootstrapping was subsequently employed in all further analyses. 


\section{MTMM CFA}

The same MTMM approach to CFA employed in Study 1was used, again involving the testing of four models: (1) CT, (2) correlated trait-correlated method, (3) correlated trait-uncorrelated method, and (4) CTCU. Similar to Study 1, improper solutions occurred for the correlated trait-correlated method and correlated traituncorrelated method. Both displayed negative variances and were consequently disregarded. The CT and CTCU models both yielded proper solutions, and displayed a good fit to the data (see Table 1). Standardized factor loadings ranged between 0.70 and 0.81 (CT model) and between 0.64 and 0.81 (CTCU model). The intertrait correlations ranged between 0.33 and 0.68 (CT model) and between 0.32 and 0.60 (CTCU model). The uniqueness correlations for the CTCU model ranged between .05 and .19. All factor loadings, modification indices, and standardized residuals were within acceptable limits (Hair et al., 1998). Inspection of the correlated error variances in the CTCU model revealed significant correlations with one another $(p<.05)$.

\section{Alternative Models}

Although results support the CTCU model and the interfactor correlation between external visual imagery and internal visual imagery was only .38 , the data were reanalyzed to verify that external visual imagery and internal visual imagery should be assessed separately. A two-trait factor model (visual imagery and kinesthetic imagery) was specified in which the four items assessing external visual imagery and four items assessing internal visual imagery were all forced onto the same factor to assess visual imagery. The four kinesthetic items remained together on the second trait factor to separately assess kinesthetic imagery. As can be seen in Table 3, results revealed a poor fit to the data when external visual imagery and internal visual imagery were forced onto the same factor.

Although the correlation between kinesthetic imagery and external visual imagery was .39, the correlation between kinesthetic imagery and internal visual

\section{Table 3 MIQ-3 Goodness-of-Fit Indices for the Correlated Trait- Correlated Uniqueness (CTCU) Model and Alternate Models Using CTCU CFA (Study 2)}

\begin{tabular}{lcccccc}
\hline Model & $\chi^{2}$ & $\boldsymbol{d f}$ & TLI & CFI & SRMR & $\begin{array}{c}\text { RMSEA } \\
\mathbf{( 9 0 \% ~ C I )}\end{array}$ \\
\hline CTCU & $75.12^{*}$ & 39 & .97 & .98 & .04 & $.05(.03-.07)$ \\
CTCU VI + KI & $369.13^{*}$ & 41 & .71 & .82 & .11 & $.15(.13-.16)$ \\
CTCU IVI, KI + EVI & $243.66^{*}$ & 41 & .82 & .88 & .07 & $.12(.10-.13)$ \\
\hline
\end{tabular}

Note. The CTCU is the correlated trait-correlated uniqueness model with external visual imagery, internal visual imagery, and kinesthetic imagery all as separate factors. The CTCU VI + KI is the correlated trait-correlated uniqueness model with external and internal visual imagery perspectives on one factor and kinesthetic imagery on the other. The CTCU IVI, KI + EVI is the correlated traitcorrelated uniqueness model with internal visual and kinesthetic imagery on one factor and external visual imagery on the other.

$* p<.001$. 
imagery was strong $(r=.60)$. When describing the process of imaging from an internal perspective, some researchers have included characteristics of kinesthetic imagery in their definition, such as "experiencing [the] sensations that might be expected in the actual situation" (e.g., Mahoney \& Avener, 1977, p. 137). Although since then there has been a call to distinguish between the two processes, we wanted to ensure each factor was assessing a different type of imagery ability. Consequently, a second alternate model was devised in which internal visual imagery and kinesthetic imagery items were forced onto one factor, and external visual imagery remained on the second factor. As can be seen in Table 3, the second two-factor alternative model also revealed a poor fit to the data, demonstrating the three-factor model to be the most appropriate.

\section{Gender Invariance}

Goodness of fit results for the five models of the gender invariance analysis are reported in Table 2. Although the change in $\chi^{2}$ was significant when the factor covariances were constrained to be equal across males and females, the change in CFI was smaller than .01. This finding, along with the nonsignificant change in $\chi^{2}$ between the other increasingly constrained models, supports the gender invariance of the final MIQ-3 model.

\section{Latent Means}

Results revealed that latent mean estimates reported by male participants did not significantly differ compared with those for females for external visual imagery $(-.195, p=.126)$, internal visual imagery $(-.136, p=.232)$, and kinesthetic imagery $(.018, p=.885)$. Similar to Study 1 , the model still displayed a good fit to the data even when the factor loadings and observed means were constrained to be equal across males and females, $\chi^{2}(102)=142.17, p=.005, \mathrm{CFI}=.98, \mathrm{TLI}=.97, \mathrm{SRMR}$ $=.05, \mathrm{RMSEA}=.03(90 \% \mathrm{CI}=.02-.05)$.

\section{Concurrent Validity}

The CFA model fit for the VMIQ-2 revealed an adequate fit to the data, $\chi^{2}(555)$ $=922.63, p<.001, \mathrm{CFI}=.93, \mathrm{TLI}=.92, \mathrm{SRMR}=.06, \mathrm{RMSEA}=.06(90 \% \mathrm{CI}=$ 0.06-0.07) and all factor loadings, modification indices, and standardized residuals were within acceptable limits (Hair et al., 1998). The measurement model was then tested as a whole. Results revealed an adequate fit to the data, $\chi^{2}(1020)=$ $1641.60, p<.001, \mathrm{CFI}=.92, \mathrm{TLI}=.90, \mathrm{SRMR}=.06, \mathrm{RMSEA}=.06(90 \% \mathrm{CI}=$ 0.06-0.07) and all factor loadings, modification indices, and standardized residuals within acceptable limits (Hair et al., 1998). Examination of the covariances revealed significant correlations between all VMIQ-2 and MIQ-3 subscales (see Table 4). Moreover, each VMIQ-2 subscale (i.e., external visual imagery, internal visual imagery, and kinesthetic imagery) correlated most highly with the same subscale measured by the MIQ-3. To further support the MIQ-3's concurrent validity, we investigated whether correlations between the VMIQ-2 and its respective subscale on the MIQ-3 (e.g., VMIQ-2 kinesthetic imagery ability and MIQ-3 kinesthetic imagery ability) were significantly greater than between other subscales. Results revealed significantly greater correlations between respective subscales (external 


\section{Table 4 Correlations Between the MIQ-3 and VMIQ-2 Subscales (Study 2)}

\begin{tabular}{lccc}
\hline & \multicolumn{3}{c}{ MIQ-3 } \\
\cline { 2 - 4 } VMIQ-2 & EVI & IVI & KI \\
\hline EVI & $0.679 * *$ & $0.554^{* *}$ & $0.259 *$ \\
IVI & $0.239 *$ & $0.629^{* *}$ & $0.351^{* *}$ \\
KI & $0.246^{*}$ & $0.533^{* *}$ & $0.706 * *$ \\
\hline
\end{tabular}

Note. EVI = external visual imagery, IVI = internal visual imagery, and $\mathrm{KI}=$ kinesthetic imagery.

$* p<.05$. ** $p<.001$.

visual imagery, $t=-2.40, p<.05$; internal visual imagery, $t=-5.92, p<.01$; and kinesthetic imagery, $t=-4.06, p<.01$ ) than correlations with other types of imagery ability (e.g., VMIQ-2 kinesthetic imagery and MIQ-3 external visual imagery). Despite the significant correlations between the VMIQ-2 and MIQ-3, these were only small to moderate in size and the largest correlation between the two questionnaires accounted for less than $50 \%$ of the shared variance. This further highlights that the MIQ-3 is tapping a different dimension of imagery ability than the VMIQ-2.

\section{Discussion}

As in Study 1, results supported a CTCU model. This is due to the significantly correlated error variances between the same methods displaying a model fit significantly better than the CT model. Consequently, and as indicated for the first time in Study 1, we conclude that a method effect exists by assessing each type of imagery ability using the same four movements.

The fact that the three-factor CTCU model provided the best fit to the data, along with the correlations between each subscale, demonstrates external visual imagery, internal visual imagery, and kinesthetic imagery ability to be separate but related constructs - a finding similar to previous research (e.g., Roberts et al., 2008). This supports the advantage of employing the MIQ-3 over the MIQ-R as a more comprehensive assessment of movement imagery ability.

The three-factor CTCU model also displayed gender invariance, demonstrating it to be a suitable multidimensional measure of movement imagery ability for both males and females. There were also no significant differences in latent mean scores due to gender, which adds to the findings of Study 1 that gender differences do not exist in movement imagery ability. Finally, significant correlations between the MIQ-3 and the VMIQ-2 demonstrate the MIQ-3's concurrent validity. The significant correlations between the same subscales on both questionnaires demonstrate that the MIQ-3, similar to the VMIQ-2, is assessing external visual imagery, internal visual imagery, and kinesthetic imagery ability. However, the fact that these correlations are only moderate in size reinforces our argument that these questionnaires are not assessing the exact same thing. Although measurement error may contribute to some of the explained variance, it is likely that some is due to the MIQ-3 assessing ease of imaging and the VMIQ-2 assessing vividness of the image. Altogether, these 
findings support the MIQ-3's capacity to assess ease of imaging external visual imagery, internal visual imagery, and kinesthetic imagery. Despite the validity and reliability evidence presented in Study 2, the MIQ-3 is in its infancy and further validation is warranted to ensure it is separately assessing external visual imagery and internal visual imagery ability. One way is to investigate the predictive validity of the different visual imagery perspectives measured by the MIQ-3 with an outcome that should be predicted differently by each imagery perspective (e.g., observational learning). To address this issue a third study was conducted.

\section{Study 3}

The acts of imagining and observing actions have been shown to share cognitive and neural processes with motor performance. For example, both imagery and observation engage a partially shared set of brain regions with movement execution (for meta-analysis and reviews, see Grèzes \& Decety, 2001; Jeannerod, 2001). Secondly, both imagery and observational learning (OL) serve cognitive (e.g., improve skills and strategies), and motivational (e.g., maintain focus and concentration) functions (Cumming, Clark, Ste-Marie, McCullagh, \& Hall, 2005; Hall, Mack, Paivio, \& Hausenblas, 1998). Furthermore, an association has been found between higher levels of imagery ability and more frequent OL (e.g., Rymal \& Ste-Marie, 2009; Williams et al., 2011) with previous research even predicting OL use to establish predictive validity of imagery ability questionnaires (Williams \& Cumming, 2012). Consequently, the MIQ-3 should be able to predict OL, and testing this hypothesis formed the purpose of Study 3. Moreover, comparing the MIQ-3 and VMIQ-2's predictive capacity would be able to further investigate which characteristic of imagery ability (i.e., ease or vividness) was a stronger predictor of OL use.

Although both visual imagery and observation can be performed from either an internal (first person) or external (third person) perspective, OL is usually done from an external perspective. For example, athletes may watch a coach correctly perform a particular training exercise that they will later execute. The same perceptual features would be seen when imaging this scene from an external visual imagery perspective (White \& Hardy, 1995). With these parallels drawn between OL and external visual imagery, White and Hardy (1995) stated that "visual models could be considered as external visual images of a skill, as they are viewed from a third-person perspective" (p. 171). These findings reinforce the need for separate measurement of external visual imagery ability and internal visual imagery ability. We postulate that external visual imagery assessed by the MIQ-3 and VMIQ-2 should predict OL, regardless of its function, to a greater extent than internal visual imagery and kinesthetic imagery. Due to the exploratory nature of comparing ease of imaging and vividness at predicting OL, we do not hypothesize that one is a stronger predictor than the other.

\section{Method}

\section{Participants}

Ninety-seven participants (male $=39$; female $=58$ ) with a mean age of 19.54 years $(S D=1.06)$ and no previous imagery training took part in Study 3. Participants 
were all athletes capable of physically performing the four MIQ-3 movements, and represented a combination of team $(n=57)$ and individual $(n=40)$ sports. The majority of athletes were recruited from soccer $(n=24)$, track and field $(n=$ $9)$, road running $(n=8)$, and swimming $(n=8)$. Participants represented a variety of competitive levels (recreational $=25$, club $=64$, regional $=6$, and elite $=2$ ) and had gained an average of 7.91 years $(S D=3.77)$ playing experience.

\section{Measures}

Demographic Information. Participants provided information regarding their age, gender, sport, competitive level, and years of playing experience.

Movement Imagery Questionnaire-3 (MIQ-3). The same MIQ-3 used in Study 2 was distributed in Study 3. In the current study, the MIQ-3 demonstrated good internal reliability for all three subscales, with CR values being .70 or above (external visual imagery $=.89$, internal visual imagery $=.81$, and kinesthetic imagery $=.89$ ) and AVE values being .50 or above (external visual imagery $=.66$, internal visual imagery $=.51$, and kinesthetic imagery $=.67$ ).

Vividness of Movement Imagery Questionnaire-2 (VMIQ-2). The same VMIQ-2 used in Study 2 was distributed in Study 3 and similarly it was reversed scored for clarity. In the current study, the VMIQ-2 demonstrated good internal reliability for each subscale with CR values of .93 (external visual imagery), .92 (internal visual imagery), and .93 (kinesthetic imagery), and AVE values of .54 (external visual imagery), .50 (internal visual imagery), and .53 (kinesthetic imagery).

Functions of Observational Learning Questionnaire (FOLQ). The FOLQ (Cumming, Clark, Ste-Marie, McCullagh, \& Hall, 2005) is a 17-item questionnaire designed to measure the frequency of an individual's use of OL. Items represent one of the three functions of athlete OL use. These are skills (e.g., I use observational learning to change how I perform a skill), strategy (e.g., I use observational learning to determine how a strategy will work in an event/game), and performance (e.g., I use observational learning to learn how to be focused during a challenging situation). Frequency of use is rated on a 7-point Likert-type scale ranging from 1 (rarely) to 7 (often). The FOLQ is a valid and reliable method of assessing an individuals' functional use of OL (Cumming et al., 2005). In the current study, the FOLQ demonstrated very good internal reliability for all three subscales, with CR values being .70 or above (skill $=.94$, strategy $=.90$, and performance $=.93$ ) and AVE values being .50 or above (skill $=.68$, strategy $=.58$, and performance $=.63$ ).

\section{Procedures}

The procedures were identical to those of Study 2 with the exception that all participants completed the VMIQ-2 and the FOLQ.

\section{Data Analysis}

To assess the predictive validity of the MIQ-3 and the VMIQ-2, three hierarchical multiple regressions were conducted to examine how accurately the three subscales 
from each questionnaire could predict each of the three FOLQ subscales. To control for age, gender, sport type, competitive level, and years of playing experience, these variables were entered into the first step of each regression. Because none of these variables significantly predicted skill, strategy, or performance OL, they were removed from subsequent analysis. The MIQ-3 and VMIQ-2 subscales were therefore entered in Step 1 to directly compare which were the stronger predictors of OL. Results of all three regressions are presented in Table 5.

\section{Table 5 Regression Analysis for MIQ-3 Imagery Ability Predicting Observational Learning Use}

\begin{tabular}{|c|c|c|c|c|}
\hline & $\Delta \boldsymbol{R}^{2}$ & $\beta$ & $t$ & $p$ \\
\hline \multicolumn{5}{|l|}{ Skill observational learning use } \\
\hline Step 1 & $.163 * *$ & & & \\
\hline MIQ-3 External visual imagery & & .39 & 2.82 & .006 \\
\hline MIQ-3 Internal visual imagery & & -.06 & -0.38 & .703 \\
\hline MIQ-3 Kinesthetic imagery & & .15 & 0.98 & .331 \\
\hline VMIQ-2 External visual imagery & & -.19 & -1.35 & .182 \\
\hline VMIQ-2 Internal visual imagery & & -.14 & -0.99 & .323 \\
\hline VMIQ-2 Kinesthetic imagery & & .22 & 1.47 & .144 \\
\hline \multicolumn{5}{|l|}{ Strategy observational learning use } \\
\hline Step 1 & $.146^{*}$ & & & \\
\hline MIQ-3 External visual imagery & & .44 & 3.17 & .002 \\
\hline MIQ-3 Internal visual imagery & & -.06 & -0.37 & .709 \\
\hline MIQ-3 Kinesthetic imagery & & -.03 & -0.16 & .871 \\
\hline VMIQ-2 External visual imagery & & -.06 & -0.44 & .663 \\
\hline VMIQ-2 Internal visual imagery & & .07 & 0.51 & .609 \\
\hline VMIQ-2 Kinesthetic imagery & & -.03 & -0.22 & .825 \\
\hline \multicolumn{5}{|c|}{ Performance observational learning use } \\
\hline Step 1 & $.225 * * *$ & & & \\
\hline MIQ-3 External visual imagery & & .15 & 1.14 & .257 \\
\hline MIQ-3 Internal visual imagery & & -.06 & -0.31 & .754 \\
\hline MIQ-3 Kinesthetic imagery & & .48 & 3.30 & .001 \\
\hline VMIQ-2 External visual imagery & & -.04 & -0.31 & .759 \\
\hline VMIQ-2 Internal visual imagery & & .15 & 1.13 & .261 \\
\hline VMIQ-2 Kinesthetic imagery & & -.45 & -1.01 & .316 \\
\hline
\end{tabular}

$* p<.05 . * * p=.010$. $* * * p<.001$. 


\section{Results}

\section{Regression Analysis}

Skill OL. Results revealed a significant overall model when predicting skill OL use, $F(6,93)=3.02, p=.010$, accounting for $16 \%$ of the variance (adj $R^{2}=.11$ ). Inspection of the beta weights revealed that only MIQ-3 external visual imagery significantly predicted skill OL use $(\beta=.39, t=2.82, p=.006)$. None of the other imagery ability subscales significantly predicted skill OL use.

Strategy OL. A significant overall model was found when predicting strategy OL use, $F(6,93)=2.65, p=.020$, accounting for $15 \%$ of the variance $\left(\operatorname{adj} R^{2}=.09\right)$. Similarly to when predicting skill OL, inspection of the beta weights revealed that only MIQ-3 external visual imagery significantly predicted strategy OL use $(\beta=$ $.44, t=3.17, p=.002$ ). None of the other imagery ability subscales significantly predicted skill OL use.

Performance OL. When predicting performance OL use a significant overall model was also found, $F(6,93)=4.49, p<.001$, accounting for $23 \%$ of the variance $\left(\operatorname{adj} R^{2}=.17\right)$. Beta weight inspection revealed that only MIQ-3 kinaesthetic imagery significantly predicted performance OL use $(\beta=.48, t=3.30, p=.001)$. None of the other imagery ability subscales significantly predicted skill OL use.

Multicollinearity diagnostics revealed that values were within acceptable limits based on the recommendations of Belsley, Kuh and Welsch, (1980).

\section{Discussion}

Results of Study 3 support the predictive validity of the MIQ-3 by the positive predictions found for cognitive and motivational functions of OL. Similarly to previous research, results demonstrate that individuals with higher levels of ease of imaging report more frequent OL. These relationships depended on the type of ease of imaging measured by the MIQ-3. In partial support of our hypothesis, external visual imagery positively predicted skill OL and strategy OL. Internal visual imagery added no significant variance to the prediction of skill and strategy OL, demonstrating that external visual imagery is a better predictor of cognitive forms of OL.

Unexpectedly for performance OL, kinesthetic imagery was the only positive predictor. Given that kinesthetic imagery is a different sensory modality to OL, it appears initially surprising that this type of imagery predicted OL. However, Bandura (1997) explains that symbolic transformation is used to retain observed information as a key subprocess of OL. An individual's ability to image kinesthetically might be an advantage when it comes to internalizing what has been viewed into a representation of a movement (skill OL) or an emotion/feeling (performance OL). Therefore, these findings suggest that kinesthetic imagery might be more important than previously considered for aiding the use of OL.

A surprising finding was that the VMIQ-2 failed to significantly predict OL use, particularly as research previously demonstrated that higher levels of imagery ability are associated with OL use (Rymal \& Ste-Marie, 2009; Williams et al., 2011). 
Nonetheless, these findings demonstrate the importance of the MIQ-3 as a measure of external visual imagery, internal visual imagery, and kinesthetic imagery ability in its own right. Research has demonstrated that observation used immediately before imaging can improve ease of imaging (Williams et al., 2011). Consequently, it can be proposed that individuals who use OL more frequently are able to retrieve this information more readily from their long-term memory to facilitate with the generation of movement images. Although they find it easier to image, they may not experience more vivid images than those who use OL less frequently. However, this is merely a suggestion, and future research should investigate the relationship between different dimensions of imagery ability and OL use.

\section{General Discussion}

Overall, the results from all three studies identified the MIQ-R and MIQ-3 to be valid and reliable measures of visual and kinesthetic movement imagery ability. The CTCU model displayed the best fit for both MIQ-R and MIQ-3 data, highlighting the method effects that occur by assessing each imagery ability trait using the same four movements, something that has been overlooked in previous attempts to validate the MIQ, MIQ-R, and MIQ-RS. Consequently, an individual's ability to image a knee lift from an external visual imagery perspective is likely to be associated with her or his ability to image a knee lift from an internal visual imagery perspective and from a kinesthetic modality, as all three include imaging the same movement (i.e., use the same method). However, the CTCU model suggests that although these method effects exist, each questionnaire item has its own method effect and the common method factor is reflected in the covariances between the items using the same method (Kenny \& Kashy, 1992). The model fit of both questionnaires was also invariant across gender, and the imagery abilities of males and females did not significantly differ according the latent means. Comparison of the three-factor MIQ-3 CTCU model with alternate two-factor models further supported the need for the MIQ-3 to separately assess external visual imagery, internal visual imagery, and kinesthetic imagery ability. Concurrent validity of the MIQ-3 was also supported through the significant correlations of external visual imagery, internal visual imagery, and kinesthetic imagery ability measured by the MIQ-3 with external visual imagery, internal visual imagery, and kinesthetic imagery ability measured by the VMIQ-2. Finally, findings supported the predictive validity of ease of imaging in relation observation, by demonstrating that subscales of the MIQ-3 helped explain athletes' use of cognitive and motivational OL.

Separately assessing each visual imagery perspective is likely to provide a more comprehensive understanding of an individual's ease of imaging and his or her capabilities of taking part in an upcoming intervention. For example, if the MIQ-R is used to screen for an internal visual imagery intervention, a researcher will not know how well the individual is specifically able to image from an internal visual imagery perspective. A visual MIQ-R score that exceeds the cutoff value may be a result of good external visual imagery and may be misleading in how effective the intervention is likely to be. By separately assessing each visual imagery perspective, the researcher is likely to be more informed of whether individuals are able to sufficiently meet the criteria of the intervention, and take the more 
appropriate action. The MIQ-3 is also beneficial in applied settings owing to its separate assessment of visual imagery perspectives. Research has demonstrated that imagery perspective ability and preferred imagery perspective, although related, are separate constructs (Callow \& Roberts, 2010). Consequently, it would appear logical for researchers, when designing an imagery intervention, to separately assess external visual imagery and internal visual imagery and take these results into consideration to try to maximize the benefits of the intervention. If an intervention uses a particular perspective, the MIQ-3 may also inform the practitioner whether they need to work with the athlete to improve their imagery ability to ensure they are able to image sufficiently.

Researchers can now access two measures that assess different aspects of movement imagery ability: the MIQ-3 and the VMIQ-2. The MIQ-3 should be preferred over the VMIQ-2 when assessing ease of imaging, or when there is a need to tightly control how movements are imaged and how recently participants have performed the movement. The VMIQ-2 would be more appropriate when assessing vividness or if space is too limited to administer the MIQ-3. When participants are unable to physically perform the MIQ-3 movements, the VMIQ-2 and the MIQ-RS are both alternatives to consider. Researchers should therefore carefully consider their research question, the testing situation, and the population to be screened, when selecting their measure of movement imagery ability.

A limitation of this research is that there is no direct comparison between the MIQ-3 and the MIQ-R. However, as test validity is a long-term process, future research should address this as well as expose the measure to other forms of validity and reliability testing. Similarly to Study 3, it would be worthwhile to investigate the predictive nature of the MIQ-3 compared with the MIQ-R for outcomes such as performance and psychological characteristics that influence performance, such as anxiety and confidence. Although the current study tested the four MTMM models advocated by Marsh (1989), future research could investigate a potential multiplicative structure by testing a direct product model and individual level method effects using the more recent MTMM approach proposed by Pohl, Steyer and Kraus (2008) to examine the model fit of the MIQ-R and MIQ-3. Research could also use nonrecursive and longitudinal models to investigate if there is a more substantive element to the cross-trait association between the same items such as a relationship between the modalities. Finally, it would be worthy to investigate whether a similar factor structure is obtained for nonathletes who are not as familiar with performing movements and monitor any changes in imagery ability when they become more acquired to physical activity.

In conclusion, the present three studies further validated the MIQ-R, and then modified it to provide a more comprehensive assessment of visual imagery ability by separately assessing external and internal visual imagery ability, in addition to kinesthetic imagery ability. Using MTMM CFA, support of a method effect existed for both the MIQ-R and the modified MIQ-3. This was due to assessing each type of imagery with the same four movements. Results in Study 2 confirmed a three-factor model in which external visual imagery, internal visual imagery, and kinesthetic imagery ability were all separate but related constructs. Finally, Study 3 began to establish the predictive validity of the MIQ-3 as a comprehensive assessment of movement imagery ability. 


\section{References}

Abma, C.L., Fry, M.D., Li, Y., \& Relyea, G. (2002). Differences in imagery content and imagery ability between high and low confident track and field athletes. Journal of Applied Sport Psychology, 14, 67-75. doi:10.1080/10413200252907743

Arbuckle, J.L. (2007). AMOS (Version 16.0). Chicago: Smallwaters Corporation. ([Computer software])

Atienza, F., Balaguer, I., \& Garcia-Merita, M.L. (1994). Factor analysis and reliability of the Movement Imagery Questionnaire. Perceptual and Motor Skills, 78, 1323-1328. PubMed doi:10.2466/pms.1994.78.3c.1323

Bandura, A. (1997). Self-efficacy: The exercise of control. New York: W.H. Freeman.

Belsley, D.A., Kuh, E., \& Welsch, R.E. (1980). Regression diagnostics: Identifying influential data and sources of collinearity. New York: John Wiley.

Bentler, P.M. (1995). EQS structural equations program manual. Encino, CA: Multivariate Software Inc.

Byrne, B.M. (2009). Structural equation modelling with AMOS: basic concepts, applications, and programming (2nd ed.). New York: Taylor and Francis.

Caliari, P. (2008). Enhancing forehand acquisition in table tennis: The role of mental practice. Journal of Applied Sport Psychology, 20, 88-96. doi:10.1080/10413200701790533

Callow, N., \& Hardy, L. (2004). The relationship between the use of kinesthetic imagery and different visual imagery perspectives. Journal of Sports Sciences, 22, 167-177. PubMed doi:10.1080/02640410310001641449

Callow, N., \& Roberts, R. (2010). Imagery research: An investigation of three issues. Psychology of Sport and Exercise, 11, 325-329. doi:10.1016/j.psychsport.2010.03.002

Callow, N., \& Waters, A. (2005). The effect of kinesthetic imagery on the sport confidence of flat-race horse jockeys. Psychology of Sport and Exercise, 6, 443-459. doi:10.1016/j. psychsport.2004.08.001

Campos, A., Pérez-Fabello, M.J., \& Gómez-Juncal, R. (2004). Gender and age differences in measured and self-perceived imaging capacity. Personality and Individual Differences, 37, 1383-1389. doi:10.1016/j.paid.2004.01.008

Cheung, G.W., \& Rensvold, R.B. (2002). Evaluating goodness-of-fit indexes for testing measurement invariance. Structural Equation Modeling, 9, 233-255. doi:10.1207/ S15328007SEM0902_5

Cumming, J., Clark, S.E., Ste-Marie, D.M., McCullagh, P., \& Hall, C. (2005). The Functions of Observational Learning Questionnaire. Psychology of Sport and Exercise, 6, 517-537. doi:10.1016/j.psychsport.2004.03.006

Cumming, J., \& Ramsey, R. (2009). Imagery interventions in sport. In S.D. Mellalieu \& S. Hanton (Eds.), Advances in applied sport psychology: A review (pp. 5-36). London: Routledge.

Cumming, J.L., \& Ste-Marie, D.M. (2001). The cognitive and motivational effects of imagery training: A matter of perspective. The Sport Psychologist, 15, 276-287 Retrieved from http://journals.humankinetics.com/tsp.

Cumming, J., \& Williams, S.E. (2012). Imagery: The role of imagery in performance. In S. Murphy (Ed.), Oxford Handbook of Sport and Performance Psychology. New York, NY: Oxford University Press.

Fourkas, A.D., Avenanti, A., Urgesi, C., \& Aglioti, S.M. (2006). Corticospinal facilitation during first and third person imagery. Experimental Brain Research, 168, 143-151. PubMed doi:10.1007/s00221-005-0076-0

Goss, S., Hall, C., Buckolz, E., \& Fishburne, G. (1986). Imagery ability and the acquisition and retention of movements. Memory \& Cognition, 14, 469-477. PubMed doi:10.3758/ BF03202518 
Gregg, M., Hall, C., \& Butler, A. (2010). The MIQ-RS: A suitable option for examining movement imagery ability. Evidence-Based Complementary and Alternative Medicine, 7(2), 249-257. PubMed doi:10.1093/ecam/nem170

Grèzes, J., \& Decety, J. (2001). Functional anatomy of execution, mental simulation, observation, and verb generation of actions: a meta-analysis. Human Brain Mapping, 12, 1-19. PubMed doi:10.1002/1097-0193(200101)12:1<1::AID-HBM10>3.0.CO;2-V

Guillot, A., Collet, C., Nguyen, V.A., Malouin, F., Richards, C., \& Doyon, J. (2009). Brain activity during visual versus kinesthetic imagery: an fMRI study. Human Brain Mapping, 30, 2157-2172. PubMed doi:10.1002/hbm.20658

Hair, J.F., Anderson, R.E., Tatham, R.L., \& Black, W.C. (1998). Multivariate data analysis. Upper Saddle River, NJ: Prentice Hall.

Hall, C.R. (2001). Imagery in sport and exercise. In R.N. Singer, H.A. Hausenblas, \& C.M. Janelle (Eds.), The handbook of sport psychology (2nd ed., pp. 529-549). New York: John Wiley \& Sons Inc.

Hall, C., Mack, D., Paivio, A., \& Hausenblas, H. (1998). Imagery use by athletes: Development of the sport imagery questionnaire. International Journal of Sport Psychology, 29, 73-89.

Hall, C.R., \& Martin, K.A. (1997). Measuring movement imagery abilities: A revision of the Movement Imagery Questionnaire. Journal of Mental Imagery, 21, 143-154.

Hall, C.R., \& Pongrac, J. (1983). Movement imagery questionnaire. London, Ontario: University of Western Ontario.

Hu, L., \& Bentler, P.M. (1999). Cutoff criteria for fit indices in covariance structure analysis: Conventional criteria versus new alternatives. Structural Equation Modeling, 6, 1-55. doi:10.1080/10705519909540118

Jeannerod, M. (2001). Neural simulation of action: A unifying mechanism for motor cognition. NeuroImage, 14, S103-S109. PubMed doi:10.1006/nimg.2001.0832

Kline, R.B. (2005). Principles and practice of structural equation modeling (2nd ed.). New York: Guilford.

Kenny,D.A., \& Kashy,D.A. (1992). Analysis of the multitrait-multimethod matrix by confirmatory factor analysis. Psychological Bulletin, 112, 165-172. doi:10.1037/0033-2909.112.1.165

Lequerica, A., Rapport, L., Axelrod, B.N., Telmet, K., \& Whitman, R.D. (2002). Subjective and objective assessment methods of mental imagery control: Construct validation of self-report measures. Journal of Clinical and Experimental Neuropsychology, 24, 1103-1116. PubMed doi:10.1076/jcen.24.8.1103.8370

Lorant, J., \& Nicholas, A. (2004). Validation de la traduction Française du Movement Imagery Questionnaire-Revised (MIQ-R). Science \& Motricite, 53, 57-68. doi:10.3917/ sm.053.0057

MacCallum, R.C. (2003). Working with imperfect models. Multivariate Behavioral Research, 38, 113-139 10.1207/S15327906MBR3801_5. doi:10.1207/S15327906MBR3801_5

Mahoney, M.J., \& Avener, M. (1977). Psychology of the elite athlete: An exploratory study. Cognitive Therapy and Research, 2, 135-141. doi:10.1007/BF01173634

Malouin, F., Richards, C.L., Jackson, P.L., \& Doyon, J. (2010). Motor imagery for optimizing the reacquisition of locomotor skills after cerebral damage. In A. Guillot \& C. Collet (Eds.), The neurophysiological foundations of mental and motor imagery (pp. 161-176). Oxford, UK: Oxford University Press.

Mardia, K.V. (1970). Measures of multivariate skewness and kurtosis with applications. Biometrika, 57, 519-530. doi:10.1093/biomet/57.3.519

Markland, D. (2007). The golden rule is that there are no golden rules: A commentary on Paul Barrett's recommendations for reporting model fit in structural equation modelling. Personality and Individual Differences, 42, 851-858. doi:10.1016/j.paid.2006.09.023

Marsh, H.W. (1989). Confirmatory factor analysis of multitrait-multimethod data: Many problems and a few solutions. Applied Psychological Measurement, 13, 335-361. doi:10.1177/014662168901300402 
Marsh, H.W., \& Grayson, D. (1995). Latent variable models of multitrait-multimethod data. In R.H. Hoyle (Ed.), Structural equation modeling: Concepts issues and applications (pp. 177-198). Thousand Oaks, CA: Sage.

Martin, K.A., Moritz, S.E., \& Hall, C. (1999). Imagery use in sport: A literature review and applied model. The Sport Psychologist, 13, 245-268 Retrieved from http://journals. humankinetics.com/tsp.

Monsma, E.V., Short, S.E., Hall, C.R., Gregg, M., \& Sullivan, P. (2009). Psychometric properties of the revised Movement Imagery Questionnaire (MIQ-R). Journal of Imagery Research in Sport and Physical Activity, 4, 1-12. doi:10.2202/1932-0191.1027

Page, S.J. (2010). An overview of the effectiveness of motor imagery after stroke: A neuroimaging approach. In A. Guillot \& C. Collet (Eds.), The neurophysiological foundations of mental and motor imagery (pp. 145-159). Oxford, UK: Oxford University Press.

Pohl, S., Steyer, R., \& Kraus, K. (2008). Modelling method effects as individual causal effects. Journal of the Royal Statistical Society. Series A, (Statistics in Society), 171, 41-63. doi:10.1111/j.1467-985X.2007.00517.x

Roberts, R., Callow, N., Hardy, L., Markland, D., \& Bringer, J. (2008). Movement imagery ability: Development and assessment of a revised version of the vividness of movement imagery questionnaire. Journal of Sport \& Exercise Psychology, 30, 200-221 Retrieved from http://journals.humankinetics.com/jsep. PubMed

Robin, N., Dominique, L., Toussaint, L., Blandin, Y., Guillot, A., \& Le Her, M. (2007). Effects of motor imagery training on service return accuracy in tennis: the role of imagery ability. International Journal of Sport and Exercise Psychology, 2, 175-186. doi:10.1080/1612197X.2007.9671818

Ruby, P., \& Decety, J. (2001). Effect of subjective perspective taking during simulation of action: a PET investigation of agency. Nature Neuroscience, 4, 546-550. PubMed

Rymal, A.M., \& Ste-Marie, D.M. (2009). Does Self-Modeling Affect Imagery Ability or Vividness? Journal of Imagery Research in Sport and Physical Activity, 4. doi:10.2202/1932-0191.1035

Schmitt, N., \& Stults, D.M. (1986). Methodology review: Analyses of multitrait-multimethod matrices. Applied Psychological Measurement, 10, 1-22. doi:10.1177/014662168601000101

Smith, D., Wright, C.J., Allsopp, A., \& Westhead, H. (2007). It's all in the mind: PETTLEPbased imagery and sports performance. Journal of Applied Sport Psychology, 19, 80-92. doi:10.1080/10413200600944132

Vadocz, E., Hall, C.R., \& Moritz, S.E. (1997). The relationship between competitive anxiety and imagery use. Journal of Applied Sport Psychology, 9, 241-253. doi:10.1080/10413209708406485

White, A., \& Hardy, L. (1995). Use of different imagery perspectives on the learning and performance of different motor skills. The British Journal of Psychology, 86, 169-180. PubMed doi:10.1111/j.2044-8295.1995.tb02554.x

Williams, S.E., \& Cumming, J. (2011). Measuring athlete imagery ability: The Sport Imagery Ability Questionnaire. Journal of Sport \& Exercise Psychology, 33, 416-440 Retrieved from http://journals.humankinetics.com/jsep. PubMed

Williams, S.E., \& Cumming, J. (2012). Athletes' ease of imaging predicts their imagery and observational learning use. Psychology of Sport and Exercise, 13, 363-370 10.1016/j. psychsport.2012.010. doi:10.1016/j.psychsport.2012.01.010

Williams, S.E., Cumming, J., \& Edwards, M.G. (2011). The functional equivalence between movement imagery, observation, and execution influences imagery ability. Research Quarterly for Exercise and Sport, 82, 555-564. PubMed doi:10.5641/0270 $13611 X 13275191444224$

Manuscript submitted: September 13, 2011

Revision accepted: June 13, 2012 